\title{
Uma nova espécie de Glaucidium \\ (Aves, Strigidae) da Amazônia.
}

Jacques Vielliard 1

\begin{abstract}
A new species of Pygmy-Owl, genus Glaucidium, is described from the Amazonian forest. It is separated from G. minutissimum, now restricted to South-eastern Brazil and Central America, by its voice and shorter tail. The type-locality is from Rondônia, but the species seems widespread, with geographical races awaiting description from northern Pará, Northeastern Brazil, and possibly Central America. A detailed survey of this overlooked canopy-living Pygmy-Owl is under way.
\end{abstract}

\section{HISTÓRICO}

O cabore Glaucidium minutissimum foi coletado na Bahia em 1816 pelo Príncipe Maximilian zu Wied-Neuwied que o descreveu sob o nome Strix minutissima em 1830. Porém, o primeiro nome atribuído a esta espécie durante quase um século foi o Strix pumila de Temminck (1821), que é um homônimo júnior do Strix pumila de Lichtenstein (1818), que era baseado simplesmente nas descrições do "Cabure" por Azara e da "Chouette" por Levaillant; a referência à Azara já tinha servido para nomear $G$. brasilianum e a descrição de Levaillant certamente refere-se à outra espécie, tornando o nome de Lichtenstein um sinônimo júnior de taxa diferentes. Assim, prevalece o nome de Wied que baseia-se nos primeiros exemplares conservados até hoje: 2 síntipos do AMNH (GREENWAY, 1978).

A partir desta data, raros exemplares apareceram esporadicamente em coleções realizadas desde os estados de São Paulo e Mato Grosso até o México. A distribuição geográfica continua até hoje pouco documentada (Fig. 1), mas várias subespécies foram descritas da América Central. Todavia, a grande variabilidade de coloração e a falta de diagnose segura entre os Glaucidium neotropicais deixaram claro a necessidade de uma revisão baseada na análise do canto, que representa o critério de reconhecimento espec ${ }^{\wedge}$ co, particularmente funcional em aves noturnas.

O primeiro que conseguiu O ivar e identificar o canto de $G$. "minutissimum" foi o saudoso Paul Schwartz: em $\left.\right|^{t}$ bo Grande (El Palmar, Bolívar, Venezuela, Fig. 1a) ele gravou um cantor que nãoł hnseguiu identificar em 04/07/66, mas coletou um macho ( $n$ ㅇ 5727) de canto idêntico em 28/04/67 e gravou um outro exemplar em 02/07/68; a cópia dessas gravaçōes, que recebi através do LNS, mostra tratar-se da mesma estrutura de canto que foi gravada ulteriormente no Peru amazônico (Fig.

1. UNICAMP, Depto. Zoologia, Cx. P., 6109, 13081 Campinas - SP 
1b, Manu, 31/07/75, J. WESKE in HARDY et al . 1980; Coffey 1981; Parker 1985) e, por mim, em Rondônia (Ji-Paraná [Fig. 1c], 14/04/83, in HARDY et al. 1989; Presidente Médici, 16/04/83, coletado macho ZUEC 1194; Samuel [Fig. 1d], 09 e 16/10/88) e no Pará (Carajás [Fig. 1e], 14/07/87; Cachoeira Porteira [Fig. 1f], 23/11/87). A frase deste canto é uma série de notas em "staccato". Um cantor deste tipo foi também gravado e coletado na mata atlântica do Nordeste por A. G. Coelho (UFPE).

A primeira edição do disco ARA-6 (HARDY et al. 1980) trazia também uma gravação atribuída a esta espécie e realizada no México por J. Arvin (Gomez Farias, Tamaulipas, 23/06/78, Fig. 1g), que era totalmente diferente; um outro exemplo desta voz é apresentado no cassete ARA-16 (HARDY et al. 1989) também do México (Naranjo, San Lui Potosi, 05/05/69, B. Coffey; Fig. 1h). Faltava então documentar a voz da população do Sudeste brasileiro, o que consegui em 10 e 11/08/84 (São Sebastião, São Paulo, macho ZUEC 1335 obtido finalmente na madrugada do dia 12); para minha maior surpresa, este canto (publicado em HARDY et al. 1989) é idêntico aos registros do México e consiste em frases de duas notas iguais bem espaçadas. Esta mesma voz foi gravada em Ubatuba (São Paulo, 11/08/84, E, Willis; Fig. 1i) e ouvida por L. Gonzaga (com. pess.) usando nossa gravação no norte do Espírito Santo (Conceição da Barra; Fig. 1j) e sul da Bahia (Fig. 1k: Monte Pascoal, Porto Seguro e Una) em outubro de 1986.

\section{DISTRIBUIÇÃO}

Além das localidades supra-citadas e dos exemplares examinados (Tab. I), existem várias menções cujas identificações não tivemos ainda a possibilidade de verificar, mas que suplementam o mapa da distribuição atualmente conhecida (Fig. 1).

Em primeiro lugar, temos os dois síntipos que Wied coletou no "interior da Bahia", o que deve ser entendido como depois dele ter-se afastado da costa, mas ainda na zona da mata, provavelmente no baixo Jequitinhonha (perto do k da Fig. 1), e não no centro árido daquele estado. Falta comparar o material de São Paulo com esses tipos, mas trata-se obviamente deste taxon.

No resto da América do Sul, onde não tem outro nome disponível, as medidas e a voz indicam referir-se a outro taxon. Sua distribuição engloba a bacia amazônica e seus "anexos": a mata atlântica do Nordeste, a bacia do Orinoco e o platô das Guianas. As localidades de coletas adicionais são: Caiçara (Mato Grosso, Pelzen 1868, Fig. 11), Cajutuba (Pará, Pinto 1938, não localizado), Rio Tacutu (Roraima, ex-Rio Branco, fide PELZELN, 1868, Fig. 1m), Kamakusa (Guiana Inglesa, fide Snyder 1966, Fig. 1n), Yarinococha (Loreto, Peru, O NEIL \& PEARSON, 1974, Fig. 1o), Tambopata (Madre de Dios, Peru, PARKER, 1982, Fig. 1p), Socarré (Córdoba, Colombia, WETMORE, 1965, Fig. 1q), Rio Tacarcuna (Choc6, Colombia, HITLY \& BROWN, 1986, Fig. 1r).

Essas duas últimas localidades fazem parte da distribuição centro-americana, que parece sub-dividida em várias populações (PETERS, 1940): rarum Griscom, 1931 no leste do Panamá e sudeste da Costa Rica (RIDGELY, 1976, Fig. 1A) gri- 
Vol. 6(4), 1989

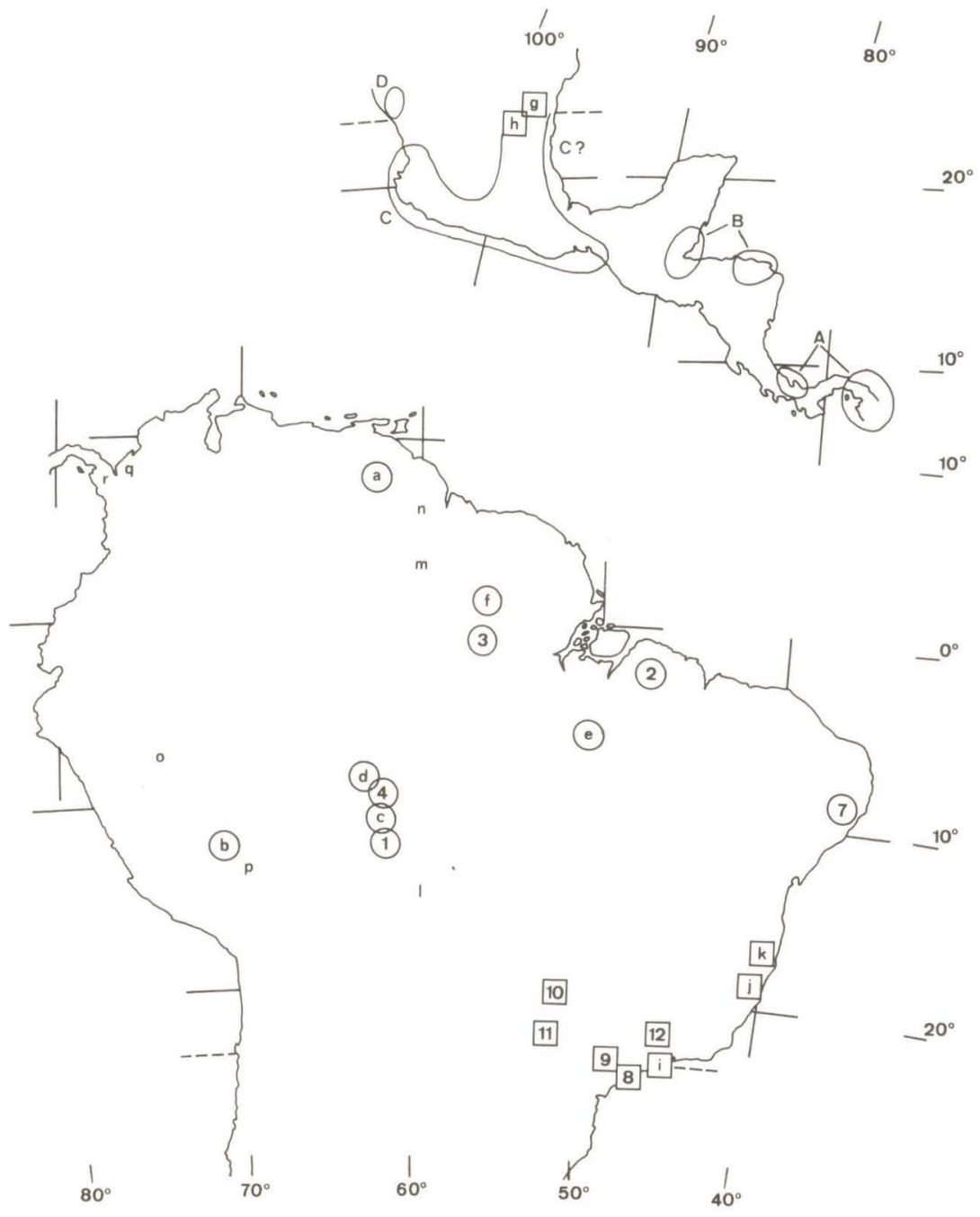

Fig. 1. Distribuiçāo do complexo Glaucidium minutissimum/hardyi Os números referem-se à Tab. I. As letras correspondem às citaçöes no texto. Quando obtivemos informaçōes sobre a voz ou as medidas que permitem uma identificação segura, o símbolo correspondendo à localidade está dentro de um quadrado (G. minutissimum) ou de um ć́rculo (G. hardyi sp. n).

seiceps Sharpe, 1875 no leste de Honduras, leste da Guatemala e Belize (Fig. 1B), palmarum NELSON, 1901 nos estados de Nayarit e Guerrero, assim como Oaxaca 
e Chiapas (DAVIS, 1972, raça não determinada; Fig. 1C) e oberholseri Moore, 1937 no estado de Sinaloa (México, Fig. 1D).

\section{Estrutura do canto}

\section{Primeiro tipo (Fig. 2A)}

A voz que gravamos em São Sebastião (SP) e que verificamos corresponder ao canto pela forte resposta territorial provocada por seu "play-back", é composta de duas notas iguais repetidas a intervalos de 3 a $5 \mathrm{~s}$. São notas puras, sem modulação, com frequência em torno de 1,3 a $1,4 \mathrm{kHz}$. A estrutura da frase é muito simples: uma nota de ca. $250 \mathrm{~ms}$, um intervalo de ca. $350 \mathrm{~ms}$, uma segunda nota idêntica; quando excitado pelo "play-back", o cantor emite às vezes uma frase similar de três notas. Nenhuma outra vocalização foi ouvida, mesmo provocando o cantor. No sudeste do Brasil, não conhecemos outra voz deste taxon.

No México, as duas gravaçöes disponíveis (vide supra) são semelhantes. O cantor de Tamaulipas apresenta uma frequência sensivelmente mais alta e um intervalo um pouco maior entre as duas notas; o de San Luiz Potosi emite frequentemente frases de três notas, também com intervalos maiores, mas na mesma frequência que em São Sebastião. Essas pequenas variações são individuais ou devidas às condições de gravação.

As outras informações sobre a voz das populações centro-americanas são meramente descritivas. Na Serra de Chiriqui (Panamá), E. Willis (com. pess.) ouviu em 1961 a mesma voz que gravou em 1984 em Ubatuba (SP). No México, I. Davis (1972) não conseguiu gravar a espécie, mas descreve bem este canto com frases de duas ou três notas e intervalos de ca. $4 \mathrm{~s}$ entre as frases. $\mathrm{Na}$ Costa Rica, segundo Slud (1964), a frase é normalmente composta de 4 notas e o intervalo entre as notas é de ca. 0,5 s. No Panamá, Ridgely (1976) descreve a frase como uma série de 3 a 5 notas, até 8 ou 9 quando o cantor é excitado. Todas essas descrições são, com variações que podem ser comportamentais ou populacionais, compatíveis com as gravações de G. minutissimum s. s. e excluem o segundo tipo de canto, em "staccato".

Segundo tipo (Fig. 2B).

O canto das populações amazônicas é um "staccato" bem diferente da vocalização anterior. O "play-back" produz uma resposta imediata, eventualmente com defesa territorial, mas sem alteração notável. Não se conhece outra vocalização e o canto do primeiro tipo nunca foi registrado nessas regiōes.

A frase é relativamente longa (1 a $3 \mathrm{~s}$ ), repetida a intervalos bem espaçados e formada pela repetição de notas praticamente iguais. Essas notas são puras, sem modulação, com frequência em torno de 1,2 a $1,3 \mathrm{kHz}$, ataque brusco e duração em torno de 30 a $40 \mathrm{~ms}$. O rítmo de emissão dessas notas é bastante rápido (11-13/s), o que produz, junto com sua intensidade constante, um efeito de "staccato" praticamente contínuo. 
Vol. 6(4), 1989

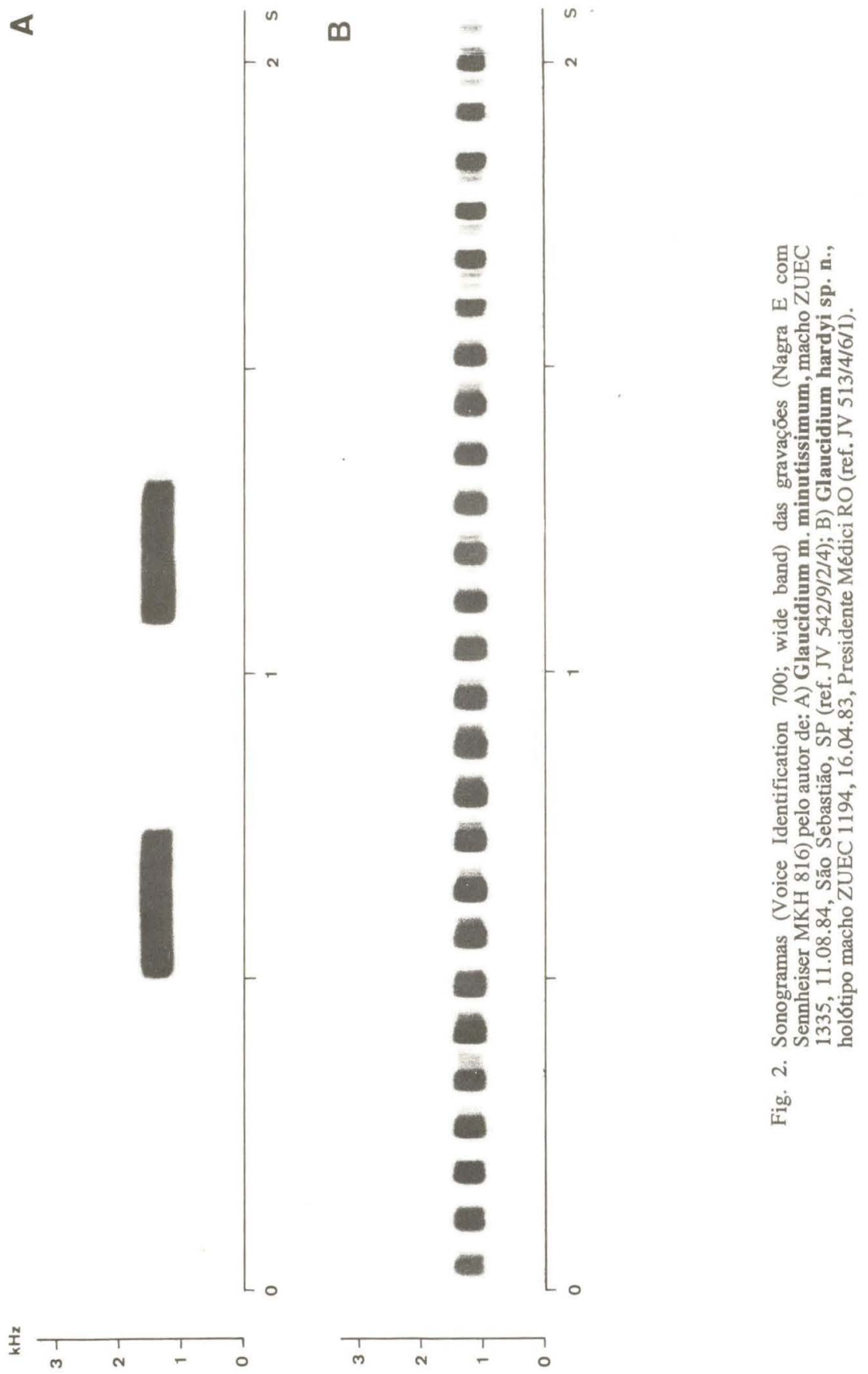


O número de notas por frase é variável: 26 a 36 para o macho de Presidente Médici, 16 a 21 para o cantor de Ji-Paraná por exemplo. Nas gravações recebidas da Venezuela, do Perú e de Pernambuco, não somente o número de notas é menor (12 a 16), mas o rítmo é um pouco mais lento (ca. 10 notas/s) e as notas sensivelmente mais longas. Além das variações individuais, que são importantes, parece existir diferenças populacionais.

\section{Comparação entre os dois tipos.}

Ambas vocalizações são cantor funcionais, com amplas variações comportamentais, individuais e aparentemente populacionais, mas com padrões estruturais totalmente distintos. $\mathrm{O}$ canto é usado pela maioria das aves e particularmente pelas corujas como sinal de reconhecimento específico e esses dois tipos de canto correspondem obviamente a duas espécies biologicamente distintas. A repartição atualmente conhecida desses dois taxa é alopátrica, mas os limites são ainda mal definidos e pode-se suspeitar a existência do taxon amazônico na América Central.

\section{MORFOLOGIA}

A coloração de Glaucidium minutissimum apresenta uma grande variabilidade e, separando os raros exemplares à nossa disposição entre os dois taxa, não conseguimos evidenciar diferenças características. $\mathrm{O}$ número de barras (4) na cauda ê o mesmo e somente é dignóstico na comparção com G. brasilianum. O grande comprimento da cauda dos exemplares do sudeste do Brasil, todavia, chamou minha atenção e reuni algumas medidas (Tab. I) que permitem definir a relação cauda/asa, um dos raros caracteres diagnósticos evidenciados por PRIGOGINE (1985) para as espécies de Glaucidium da África. Nossos dados confirmam que com uma relação cauda/asa superior a $60 \%$ minutissimum s. $s$. tem uma cauda relativamente longa e pode ser separada do taxon amazônico onde esta relação tem valores inferiores. Para as populações centro-americanas, só temos as medidas de RIDGWAY (1914): 1 macho e 1 fêmea palmarum com $64 \%$ e $65 \%$ respectivamente de relação cauda/asa estâo em conformidade com minutissimum s. $s$. mas 3 indivíduos de griseiceps-rarum mostram uma relação inferior a $60 \%$.

Apesar de faltar mais medidas padronizadas e comparações com os tipos das várias populações descritas, a necessidade de um nome para o taxon amazônico é urgente. $\mathrm{Na}$ ausência de informações provando que um dos nomes disponíveis para as populações centro-americanas possa ser aplicado a este taxon, descrevo-o como espécie. Caso comprove-se que uma das populações descritas da América Central pertence à este taxon, precisaria ainda verificar a aplicabilidade do seu nome e então transferir meu nome ao nível sub-específico. Conforme indicações de F. Novaes e A. G. Coelho sobre, respectivamente os exemplares de Cachoeira Porteira e Saltinho (veja Tab. I), estes correspondem à raças geográficas, que sugeri a eles descrever. 
Vol. 6(4), 1989

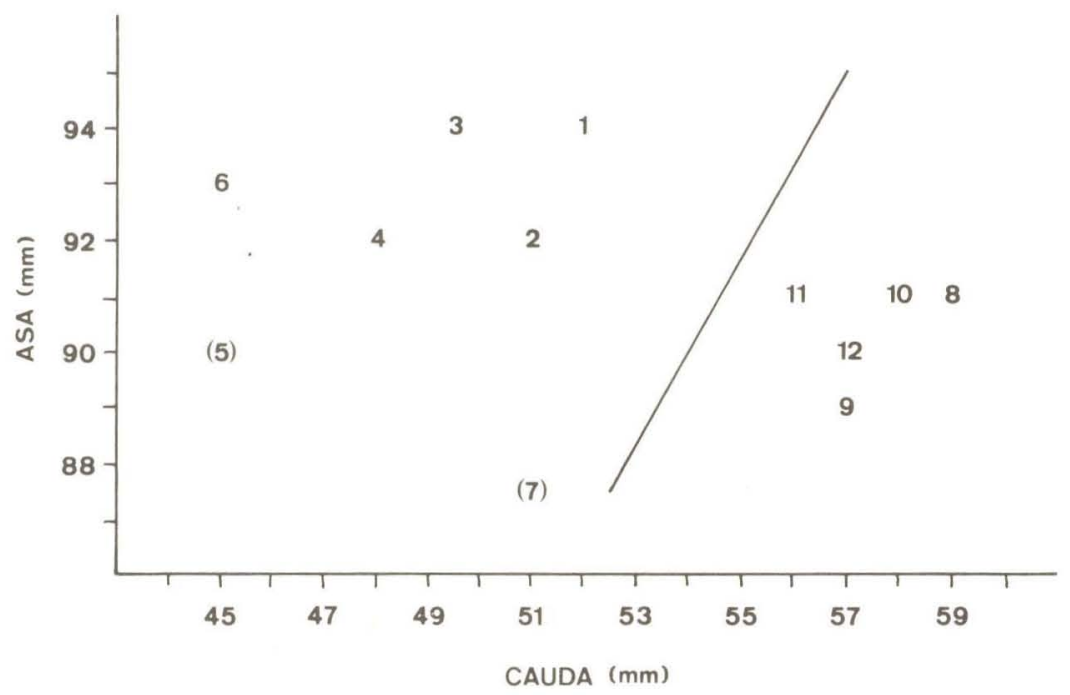

Fig. 3. Mensurações dos exemplares do complexo Glaucidium minutissimum/hardyi. Os números referem-se à Tab. I. Os exemplares 3-6 foram medidos por Fátima Cunha Lima e o exemplar 7 por Galileu M. Coelho. A linha corresponde à relação Cauda/Asa $=0,60$.

TABELA I: Exemplares do complexo Glaucidium minutissimum/hardyi examinados

№ Registro Data Localidade Asa Cauda Cauda/Asa Sexo

G. hardyi

1 ZUEC 1194 16/04/83 Pres. Médici, Ro

$\begin{array}{llll}94 & 52 & 0,55 \quad \mathrm{~m}\end{array}$

2 MZUSP 44084 25/09/59 Capim, Pa

$\begin{array}{llll}92 & 51 & 0,55 \quad \mathrm{~m}\end{array}$

3 MPEG 33105 14/07/79 Cach. Porteira, Pa $94 \quad 49,5 \quad 0,53 \quad$ f

4 MPEG 39409 25/10/86 Cach. Nazare, Ro $\quad 92 \quad 48 \quad 0,52 \quad \mathrm{~m}$

5 MPEG 39410 03/11/86 idem

6 MPEG $39411 \quad 08 / 11 / 86$ idem

7 UFPE 1017 27/09/89 Saltinho, Pe

90 - $\quad$ - $\quad \mathrm{m}$

$\begin{array}{llll}93 & 45 & 0,48 & \mathrm{~m}\end{array}$

G. minutissimum

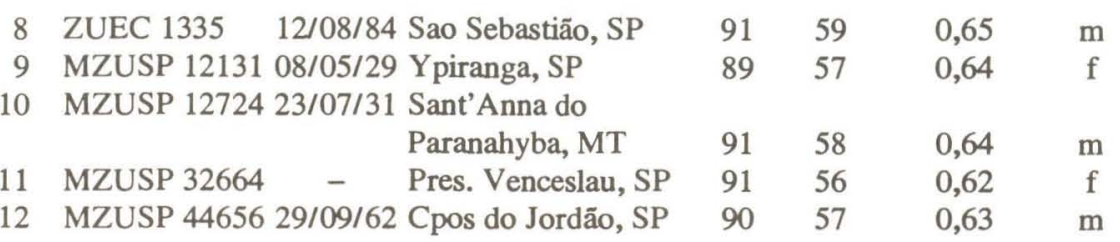


Tenho o prazer de homenagear meu colega John William Hardy, curador do Laboratório de Bioacústica do Florida State Museum, pela contribuição decisiva que ele deu ao estudo das corujas neotropicais, dando ao taxon amazônico em discussão o nome:

\section{Glaucidium hardyi sp. n.}

Holótipo: macho, 16 abril de 1983, 20 km SW Presidente Médici, Rondônia, Brasil; pele ZUEC 1194; gravação JV 513/4 no Arquivo Sonoro Neotropical; medidas: ver Tab. I; na copa da mata de terra firme; íris amarela.

Diagnose: semelhante a $G$. minutissimum, porém cauda (também com 4 barras) mais curta (relação Cauda/Asa inferior à 0,60 ) e canto distinto (ver sonogramas, Fig. 2).

Distribuição: mata amazônica, até o Orinoco e a mata atlântica do Nordeste; várias raças geográficas, a serem descritas; amplamente distribuido e localmente abundante, porém despercebido por viver nas copas e emaranhados de ciṕss.

O objetivo desta descrição preliminar é de permitir um melhor estudo do complexo G. minutissimum/hardyi e chamar a atenção sobre os problemas envolvidos. Temos em preparação um trabalho mais detalhado, com ilustrações e análises bioacústicas, mas esperamos que informações complementares sobre as populações centro-americanas estejam rapidamente disponíveis para uma avaliação mais completa e segura desses taxa. $\mathrm{O}$ aspecto mais problemático está nas relações das populações da América Central com a do Sudeste brasileiro.

\section{AGRADECIMENTOS}

Este trabalho, ainda que preliminar, é o resultado de várias expedições do autor e inúmeras colaborações. Agradeço particularmente o CNPq pelos auxílios ao Projeto "Estudo bioacústico das aves do Brasil" do qual resulta a presente publicação, a FMB pelos auxílios ao Projeto "Ornitologia do Estado de São Paulo", a Eletronorte pelas expedições em Cachoeira Porteira e Samuel, a CVRD pela expedição a Carajás, o Museu de Zoologia da Universidade de São Paulo (MZUSP, Dr. Hélio Camargo) pelo acesso à coleção, o Museu Paraense Emilio Goeldi (MPEG, Dr. Fernando Novaes e Fátima Cunha Lima) pelas informações sobre seus exemplares, a coleção da Universidade Federal de Pernambuco (UFPE, A. Galileu Coelho) sobre seu material, os funcionários do Departamento de Zoologia da Universidade Estadual de Campinas (ZUEC) pela preparação dos meus exemplares, a Library of $\mathrm{Na}$ tural Sounds (LNS, Cornell University) pelas gravaçōes fornecidas, os colegas Dr. Luiz Otávio Marcondes-Machado, Dr. Hipólito F. Paulino (in memoriam), Dr. Edwin O. Willis, A. Galileu Coelho, Wesley R. Silva e Cecília Copi pela colaboração no laboratório e no campo. 
Vol. 6(4), 1989

\section{REFERENCIAS}

COFFEY, B.B. \& L.C., 1981. Birds Songs and Calls from Southeast Peru Memphis, Tenn.

DAVIS, L.I., 1972. A field guide to the Birds of Mexico and Central America Austin, Texas.

GREENWAY, J.C., 1978. Type specimens of birds in the American Museum of Natural History. Bull. A. M. N. H. 161: 1-306.

HARDY, J.W. \& B.B. COFFEY, 1980. Voices of New World Nighbirds. ARA-6 (1 ${ }^{\text {a }}$ ed.), Florida State Museum.

HARDY. J.W., B.B. COFFEY, \& G.B. REYNARD, 1989. Voices of New World Owls. ARA-16, Florida State Museum.

HILTY, S.L.\& W.L. BROWN, 1986. A guide to the Birds of Colombia. Princeton. N.J.

O'NEILL, J.P. \& D.L. PEARSON, 1974. Estudio preliminar de las aves de Yarinococha. Publ. Mus. Hist. Nat. "Javier Prado”, Zool. 25: 1-13.

PARKER, T.A., 1982. Observations of some unususal rainforest and marsh birds in southeastern Peru. Wilson Bull. 94: 477-493.

PARKER, T.A., 1985. Voices of the Peruvian Rainforest. LNS, Ithaca, NY.

PELZELN, A. von, 1868. Zur Ornithologie Brasiliens. Wien.

PETERS, J.L., 1940. Check-list of birds of the World, 4. Cambridge, Mass.

PINTO, O.M. de O., 1938. Catálogo das Aves do Brasil. Rev. Mus. Paulista 22.

PRIGOGINE, A., 1985. Statut de quelques chevêchettes africaines et description d'une nouvelle race de Glaucidium scheffleri du Zaire. Gerfaut 75: 131-139.

RIDGELY, R.S., 1976. A guide to the Birds of Panama. Princeton, N.J.

RIDGWAY, R., 1914. The birds of North and Middle America, 6 Bull. U. S. N. M. 50.

SLUD, P., 1964. The birds of Costa Rica. Bull. A. M. N. H. 128.

SNYDER, D.E., 1966. The birds of Guyana. Salem, Mass.

WETMORE, A., 1965. Additions to the list of birds of the Republic of Colombia Q.R. f. O. 35 (sp.): 156-162. 